\title{
Decreased hospital charges and postoperative pain in septoplasty by application of enhanced recovery after surgery
}

This article was published in the following Dove Press journal:

Therapeutics and Clinical Risk Management

\begin{abstract}
Zhenpeng Liao, 1,*Wei Liao, 1,* Kai Sen Tan, ${ }^{2, *}$ Yueqi Sun, ${ }^{3}$ Aiqing Peng,' Yingxian Zhu, ${ }^{4}$ Haixin He,' Shuowei Yang,' Guangfu Xu,' Rongfei Su,' Jinyu Yao, ${ }^{3}$ Yunping Fan,' Qintai Yang, ${ }^{5}$ Haiyu Hong'

'Department of Otolaryngology, Allergy Center, The Fifth Affiliated Hospital of Sun Yat-sen University, Zhuhai, People's Republic of China; ${ }^{2}$ Department of Otolaryngology, National University of Singapore, National University Health System, Singapore, Singapore; ${ }^{3}$ Department of Otolaryngology, Otorhinolaryngology Hospital, the First Affiliated Hospital of Sun Yat-sen University, Guangzhou, People's Republic of China; ${ }^{4}$ Department of Anesthesiology, The Fifth Affiliated Hospital of Sun Yat-sen University, Zhuhai, People's Republic of China; ${ }^{5}$ Department of Otolaryngology, The Third Affiliated Hospital of Sun Yat-sen University, Guangzhou, People's Republic of China

*These authors contributed equally to this work
\end{abstract}

Correspondence: Qintai Yang

Department of Otolaryngology, The Third Affiliated Hospital of Sun Yat-sen University, No 600, Tianhe Road, Guangzhou 510630 ,

People's Republic of China

Tel +86 I37 24859848

Email yang.qt@।63.com

Haiyu Hong

Department of Otolaryngology, Allergy Center, The Fifth Affiliated Hospital of Sun Yat-sen University, No 52, Meihua East Road, Zhuhai 519020, People's

Republic of China

Tel +86 I382307 0089

Email honghy@sysu.edu.cn
Objective: Septoplasty has been the definitive treatment for nasal septum deviation, but its postoperative procedure may affect patients' quality of life. While new procedures in general surgery, such as enhanced recovery after surgery (ERAS), can speed up postoperative rehabilitations to improve quality of life, it is rarely applied in the ear-nose-throat field. This study therefore aims to evaluate the application of ERAS in patients with nasal septum deviation as a means of improving perioperative outcomes.

Materials and methods: Fifty patients with nasal septum deviation undergoing septoplasty were randomized as ERAS or control group (25 patients in both groups). Patients were investigated for outcomes including length of stay, operating time, bleeding volume, total cost, complications, and Self-Rating Anxiety Scale (SAS) and visual analog symptom score of nasal obstruction, sleep disturbance, and head facial pain.

Results: The preoperative anxiety in ERAS group (SAS 35.4 \pm 6.2 ) was lower than the control

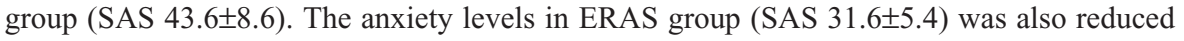
compared to the control group (SAS 38.1 \pm 10.4 ) in the 3 days postsurgery, but showed no significant difference thereafter at 7 days postsurgery. In addition, the length of stay and total cost were significantly lower for the ERAS group as well. The visual analog symptom score of nasal obstruction, sleep disturbance, and head facial pain in ERAS group were all also found to be lower than the control group. The only outcomes with no significant differences were the operation time, blood volume, and complications between the groups.

Conclusion: Our study indicated ERAS application can reduce hospital charges and postoperative pain in septoplasty, thereby improving patient quality of life and hospital expenses at the same time.

Keywords: enhanced recovery after surgery, hospital charges, nasal septum deviation, postoperative pain, septoplasty

\section{Introduction}

Nasal septal deviation is one of the common diseases of otolaryngology that can seriously affect a patient's quality of life due to its effect on nasal function like headache, nasal obstruction, hyposmia, and runny nose. ${ }^{1}$ Currently, treatment for nasal septum deviation involved septoplasty, which can sometimes result in postoperative complications such as bleeding, adhesion, and hematoma of the nasal septum. In order to reduce these postoperative complications and stabilize the nasal structure, it is conventional to pack high-expansion hemostatic sponge (Merocel, Medtronic Inc., Minneapolis, MN, USA) in nasal cavity for 48 hours. ${ }^{2}$ However, the usage of nasal filling can cause serious pain to the patients for the duration of its application postoperation. Moreover, 
removing the stuffing afterwards tends to cause bleeding and further pain, which may further affect patients' postoperation quality of life.

Enhanced recovery after surgery (ERAS) was invented and developed by a group of academic surgeons in London in $2001 .^{3}$ It is a series of effective measures applied during preoperation, intraoperation, and postoperation to reduce surgical stress and complications, which can help speed up the postoperative rehabilitation, reduce pain, and shorten hospital stay, ultimately cutting down on the overall medical cost. ${ }^{4}$ Other names are also used as fast-track surgery and enhanced recovery programs. ${ }^{5}$ The ERAS measures include the following: 1) patient preoperative education; 2) more appropriate use of anesthesia techniques, surgical techniques, and analgesic therapy for reducing surgical stress and postoperative pain and complications; and 3) strengthening of postoperative rehabilitation therapy. At present, ERAS is predominantly applied for general surgery and bone surgery, which has shown remarkable results in postoperative care. ${ }^{6,7}$ In the field of otolaryngology, postoperative complications can severely impact patient quality of life due to the effects of respiratory symptoms that may affect day-to-day operations. Therefore, ERAS application to reduce postoperative pain, shorten hospital stay, and reduce hospitalization expenses may be beneficial in improving the overall effectiveness of surgery, postoperative, care and patient quality of life in the field. The aim of this study therefore was to assess improvements in postoperative care of otolaryngology surgeries following the first ERAS application in the field.

\section{Materials and methods Subjects}

From December 2017 to February 2018, 50 cases who underwent nasal septal correction in the Department of Otolaryngology of the Fifth Affiliated Hospital of Sun Yat-sen University were selected for further clinical observation and follow-up. The patients were divided into the control group (traditional group) and the ERAS group, with 25 cases in each group. The control group consisted of 13 males and 12 females with an average age of 35.6 years, while the ERAS group consisted of 15 males and 10 females with an average age of 37.2 years. The study was approved by the Ethical Committees of the Fifth Affiliated Hospitals of Sun Yat-sen University (Approved No. of ethic committee: 2017-K135-1), and written informed consent was obtained from each subject. The patient characteristics are listed in Table 1 .
Table I Patient characteristics

\begin{tabular}{lll}
\hline & ERAS & Control \\
& group $(\mathbf{n}=\mathbf{2 5})$ & group $(\mathbf{n}=\mathbf{2 5})$ \\
\hline Age, mean $\pm S D,($ years $)$ & $37.2 \pm 10.5$ & $35.6 \pm 13.7$ \\
Sex (female:male) & $15: 10$ & $13: 12$ \\
BMI, mean $\pm S D,\left(\mathrm{~kg} / \mathrm{m}^{2}\right)$ & $21.1 \pm 2.9$ & $22.7 \pm 2.0$ \\
\hline
\end{tabular}

Abbreviations: BMI, body mass index; ERAS, enhanced recovery after surgery.

\section{Inclusion criteria}

The inclusion criteria were as follows: 1) confirmed nasal septal deviation using endoscopy and computed tomography examination; 2) patients presented with different degrees of headache, nasal congestion, recurrent nasal bleeding, hyposmia, abscess, and other clinical symptoms; 3) no improvement of symptoms and assessment following conservative treatment.

\section{Exclusion criteria}

Exclusion criteria were as follows: sinusitis, coagulant dysfunction; diabetics; mental abnormality; patients who are unfit or unsuitable for surgery; heart, kidney, and other important organ disorders; patients with respiratory diseases.

\section{Procedure}

Patients followed the specific implementation measures and management of ERAS during hospital stay, as described in Table 2 .

The intraoperative treatment of patients in ERAS group utilized trans-septal sutures for nasal septum, which can fix the nasal septum cartilage and repair torn mucosa. Furthermore, the nasal septal suture not only can prevent septal hemorrhage and hematoma, but also lessen patient pain and anxiety during nasal packing removal. The choice of the suture was made based on a randomized controlled clinical study by Awan and Iqbal, ${ }^{8}$ which showed that nasal septal suture was better than nasal filling in reducing postoperative pain, where there was no difference in the incidence of postoperative complications. Moreover, Hari et $\mathrm{al}^{9}$ also applied uninterrupted suture technique instead of interrupted suture technique to deal with the septum, which can be better protect torn local mucosa and prevent the postoperative complications such as bleeding and septal hematoma.

By referring to Hari et al's ${ }^{9}$ nasal septum suture method, the operation was performed with a $0^{\circ}$ nasal endoscope, and vircryl 4-0 absorbable angle needle for suture. The first needle is inserted from the left side of the nasal septum anterior to the middle turbinate to the right nasal cavity. Then, the needle is inserted from the back and downward of right nasal septum 
Table 2 Concrete implementation measures of ERAS

\begin{tabular}{lll}
\hline & ERAS group & Control group \\
\hline $\begin{array}{l}\text { Preoperative } \\
\text { ERAS education } \\
\text { Nothing Per } \text { os }^{\mathrm{b}}\end{array}$ & Done & None \\
Intraoperative & Fasting 6 hours, fasting water 2 hours & 8 hours \\
Anesthesia scheme & Local anesthesia and GA & Tracheal intubationa and GA \\
Temperature control & Supervise and avoid hypothermia & Common processing \\
Liquid control & Recude the infusion & Common processing \\
Cavity processing & Nasal septum stitching, without nasal filling \\
Postoperative & & Expansion sponge \\
Common analgesia & Oral NSAID & None \\
Early eating and drinking & 2 hours after surgery & None \\
Early off-bed activity & 2 hours after surgery & None \\
Remove nasal pecking & None & 48 hours after surgery \\
\hline
\end{tabular}

Notes: aERAS education: explaining of ERAS process and signing of informed notification. ${ }^{b}$ Nothing Per os: fasting for solids and liquids. ' $\mathrm{GA}$ : common processing.

Abbreviations: ERAS, enhanced recovery after surgery; GA, general anesthesia; NSAID, nonteroidal anti-inflammatory drug.

to the left nasal cavity. After that, the " $Z$ " zigzag pattern is used in this method to make an uninterrupted suture. Finally, the septum incision was closed by tying the fixed suture.

\section{Assessment}

The assessment of ERAS effect is performed based on length of stay (days), operating time (minutes), bleeding volume $(\mathrm{mL})$, total cost (Dollars), complications, visual analog symptom score (VAS) which includes nasal obstruction, sleep disturbance, head facial pain (scored on a scale of 0-10 where patients mark their severity of the symptoms on the reverse side of scale and the physician evaluates the score indicated by the patients ${ }^{10}$ ), and perioperative anxiety evaluation on a self-rating anxiety scale (SAS; the boundary value of SAS standard score is 50, a score of 50-59 is for mild anxiety, 60-69 is for moderate anxiety, and 70 is for severe anxiety $\left.{ }^{11}\right)$.

\section{Hospital discharge criteria}

A VAS of each index (nasal obstruction, sleep disturbance, head facial pain) at $<3$ was set as a criteria for discharge.

\section{Statistical method}

All data were assessed using SPSS 22.0 statistical software (IBM Corporation, Armonk, NY, USA). Measurement data (including average age, postoperative such confinement and VAS score) used $\mathrm{x} \pm \mathrm{s}$. Comparison between the 2 groups used independent-sample $t$-test. N (\%) was used for enumeration data (including gender, disease, and postoperative complications), and Fisher's exact test method or $\chi^{2}$ test was used between the 2 groups. $P<0.05$ indicates that the difference is statistically significant.

\section{Results}

Table 3 and Figures 1 and 2 show that there was no significant difference in the operation time and blood volume between the ERAS group and the control group (traditional surgical procedures). Additionally, none of the 50 patients in the 2 groups had postoperative complication. However, length of stay and total cost were significantly lower in the ERAS group than in the control group. The patients in the control group (traditional surgical procedures) generally required 5.8 days of hospitalization; while with ERAS application, the time was significantly reduced to 4.4 days. The reduction can greatly reduce hospital stay, which increases the utilization rate of beds. In terms of total hospital expenses, the application of ERAS (1,252.5 Dollars) also cost significantly less than traditional nasal septal surgery (1,333.7 Dollars), which reduces the financial burden of patients.

The preoperative anxiety in ERAS group (SAS 35.4 \pm 6.2 ) was significantly lower than that of the traditional group (SAS 43.6 \pm 8.6 ), as shown in Table 4 and Figure 3. This was due to the ERAS group having sufficient preoperative

Table 3 Analysis of control group and ERAS group

\begin{tabular}{llll}
\hline & ERAS group & Control group & $P$-value \\
\hline $\begin{array}{l}\text { Length of stay, } \\
\text { mean } \pm S D, \text { (days) }\end{array}$ & $4.4 \pm 0.5$ & $5.8 \pm 0.8$ & $<0.00 \mathrm{I}$ \\
$\begin{array}{l}\text { Operating time, } \\
\text { mean } \pm S D,(\text { minutes) }\end{array}$ & $33.4 \pm 4.1$ & $30.7 \pm 5.1$ & 0.103 \\
$\begin{array}{l}\text { Bleeding volume, } \\
\text { mean } \pm S D,(m L)\end{array}$ & $6.2 \pm 2.1$ & $6.6 \pm 2.3$ & 0.161 \\
$\begin{array}{l}\text { Total cost, mean } \pm S D, \\
\text { (Dollars) }\end{array}$ & $1,252.5 \pm 21.0$ & $1,333.7 \pm 38.1$ & $<0.00 \mathrm{I}$ \\
Complications & 0 & 0 & - \\
\hline
\end{tabular}

Notes: Statistically significant $(P<0.05)$; - indicates that not able to compare $P$ values between the two groups.

Abbreviation: ERAS, enhanced recovery after surgery. 


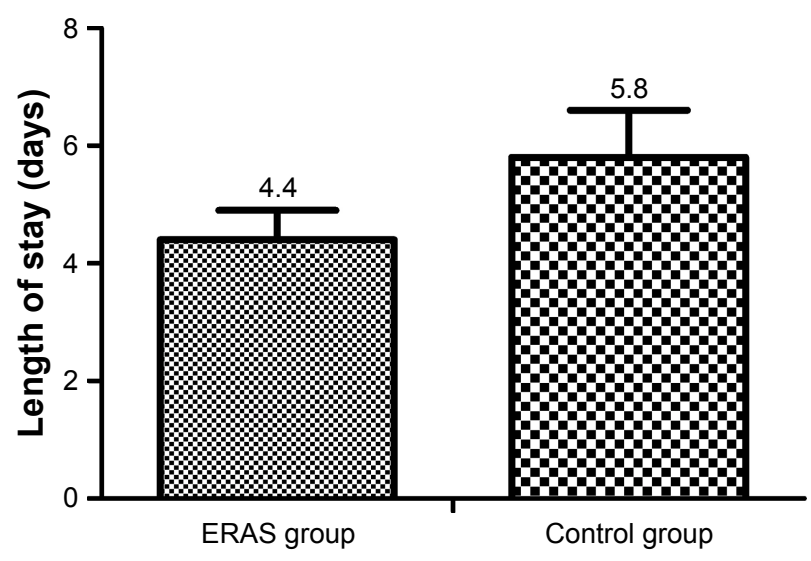

Figure I The mean hospital stay of patients in the ERAS group was shorter than the control group.

Abbreviation: ERAS, enhanced recovery after surgery.

physician-patient communication which detailed the treatment procedures. The level of anxiety in ERAS group (SAS 31.6 \pm 5.4 ) also remained lower compared to the traditional group (SAS $38.1 \pm 10.4$ ) 3 days postsurgery, possibly due to the ERAS group not using nasal cavity filling, which prevented additional postoperative pain from the nasal filling removal. Furthermore, the nasal cavity was more ventilated after surgery and sleep was more restful, and the appropriate use of painkillers during the perioperative period helped prevent mood swings that arise from postoperative pain. The anxiety index of the 2 groups showed no significant difference on the seventh day postoperation, indicating that the therapeutic effect of the 2 groups is comparable, and the application of ERAS does not affect the curative effect.

Table 5 and Figure 4 show that nasal congestion situation of patients with nasal septum is not only alleviated in the ERAS group (VAS preoperative 7.2, the first day after operation 5.1), but also met discharge standards by the third day postoperation (VAS 2.8). On the other hand, the congestion situation on the

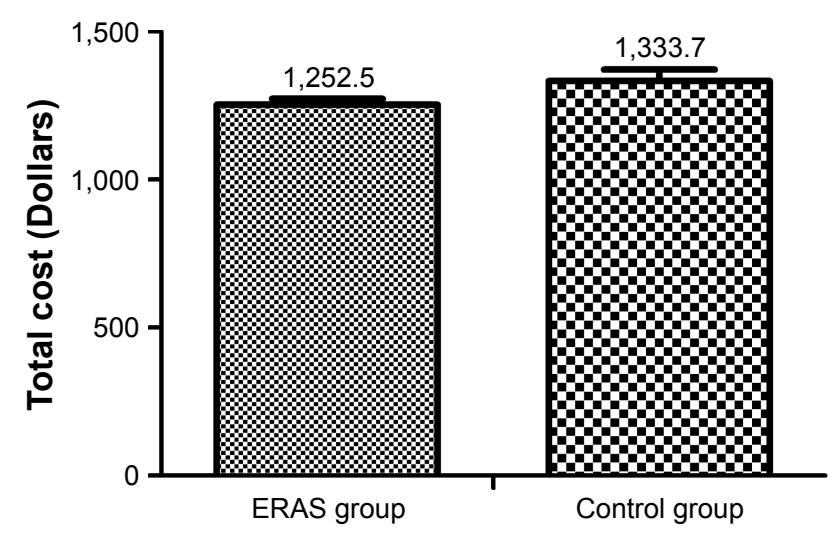

Figure 2 The mean total cost in ERAS group was lower than the control group. Abbreviation: ERAS, enhanced recovery after surgery.
Table 4 SAS score preoperation and after operation

\begin{tabular}{llll}
\hline SAS score & ERAS group & Control group & $P$-value \\
\hline Preoperation & $35.4 \pm 6.2$ & $43.6 \pm 8.6$ & $<0.001$ \\
3 days & $31.6 \pm 5.4$ & $38.1 \pm 10.4$ & 0.034 \\
7 days & $29.2 \pm 5.0$ & $31.2 \pm 9.3$ & 0.305 \\
\hline
\end{tabular}

Notes: Statistically significant $(P<0.05)$. Data presented as mean \pm SD.

Abbreviation: SAS, Self-Rating Anxiety Scale.

first (VAS 9.2) and second (VAS 9.0) day postoperation was more serious than preoperation (VAS 6.9) in the traditional group. This was due to patients only able to breathe through the mouth because of the application of postoperative nasal cavity filling, increasing the tendency to have dry or sore throat and headache. The nasal congestion in the traditional group eased starting from the third day postoperation and reached the discharge standard on the fifth day postoperation (VAS 3.0) upon removal of nasal packing.

There was little impact on sleep in the ERAS group after operation. The VAS of sleep disturbance was 3.1 on the first day and 2.6 on the second day postoperation. Therefore, the application of ERAS ensured patients to get good rest, improved the clinic experience, and promoted postoperative recovery. In contrast, sleep was highly affected in the traditional group, with a VAS of 6.7 on the first day postoperation due to the nasal filling. The VAS score was at 6.5 on the first day and dropped to 2.8 on the fourth day postoperation.

The ERAS group had an even greater improvement in terms of head and/or facial pain. The VAS score of pain was 3.5 on the first day postoperation, while the control group scored 6.5. That meant the pain of the control group was almost twice that of the ERAS group. Furthermore, the pain

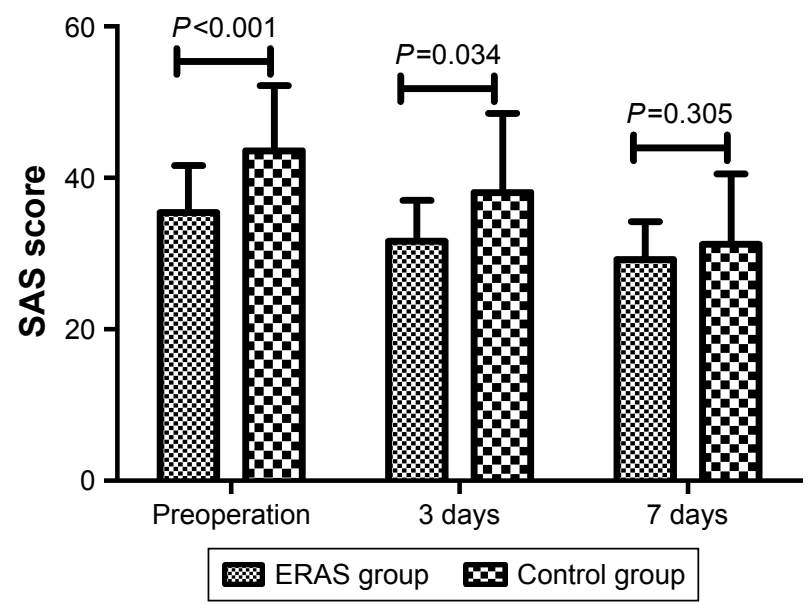

Figure 3 The SAS score in ERAS group was lower than the control group in preoperation and 3 days postoperation, but showed no significant difference compared with those of 7 days postoperation.

Abbreviations: ERAS, enhanced recovery after surgery; SAS, Self-Rating Anxiety Scale. 
Table 5 VAS score preoperation and after operation

\begin{tabular}{|c|c|c|c|c|c|c|}
\hline VAS & Preoperation & I day & 2 days & 3 days & 4 days & 5 days \\
\hline \multicolumn{7}{|l|}{ Nasal obstruction } \\
\hline ERAS group & $7.2 \pm 1.5$ & $5.1 \pm 1.2 *$ & $3.5 \pm 1.2 *$ & $2.8 \pm 0.9 *$ & & \\
\hline Control group & $6.9 \pm 1.5$ & $9.2 \pm 0.7$ & $9.0 \pm 0.8$ & $5.5 \pm 1.7$ & $4.1 \pm 1.2$ & $3.0 \pm 1.1$ \\
\hline \multicolumn{7}{|l|}{ Sleep disturbance } \\
\hline ERAS group & $1.6 \pm 1.2$ & $3.1 \pm 1.4^{*}$ & $2.6 \pm 1.3^{*}$ & & & \\
\hline Control group & $1.9 \pm 1.5$ & $6.7 \pm 2.4$ & $6.5 \pm 2.3$ & $4.3 \pm 1.0$ & $2.8 \pm 1.1$ & \\
\hline \multicolumn{7}{|l|}{ Head facial pain } \\
\hline ERAS group & 0 & $3.5 \pm 1.3 *$ & $2.4 \pm 0.9 *$ & $2.0 \pm 0.8^{*}$ & & \\
\hline Control group & 0 & $6.5 \pm 2.2$ & $6.0 \pm 2.0$ & $3.9 \pm 0.9$ & $3.1 \pm 0.9$ & $2.5 \pm 0.6$ \\
\hline
\end{tabular}

Notes: Data presented as mean \pm SD. *Statistical differences between ERAS group and Control group $(P<0.05)$.

Abbreviations: ERAS, enhanced recovery after surgery; VAS, visual analog symptom score.

continued to decrease in the ERAS group on the second day postoperation, which met the discharge standard with a VAS of 2.4, possibly aided by the appropriate use of painkillers. On the other hand, the pain in the traditional group showed almost no significant change on the second day postoperation (VAS 6.0), which was largely related to the pain caused by the nasal filling. The traditional group's pain VAS score only began to reduce on the third (3.9), fourth (3.1), and fifth (2.5) days postsurgery after the removal of the nasal packing.

\section{Discussion}

Septal deviation is a common and multifactorial clinical symptom that has a significant impact on the quality of life through its many complications that arise as the disease progresses. ${ }^{12}$ This is usually caused by a trauma during the growth period and genetic factors. ${ }^{13}$ The most common treatment for nasal septum deviation is through traditional nasal endoscopic septoplasty, which can relieve nasal congestion and hemorrhage, but has disadvantages including postoperative

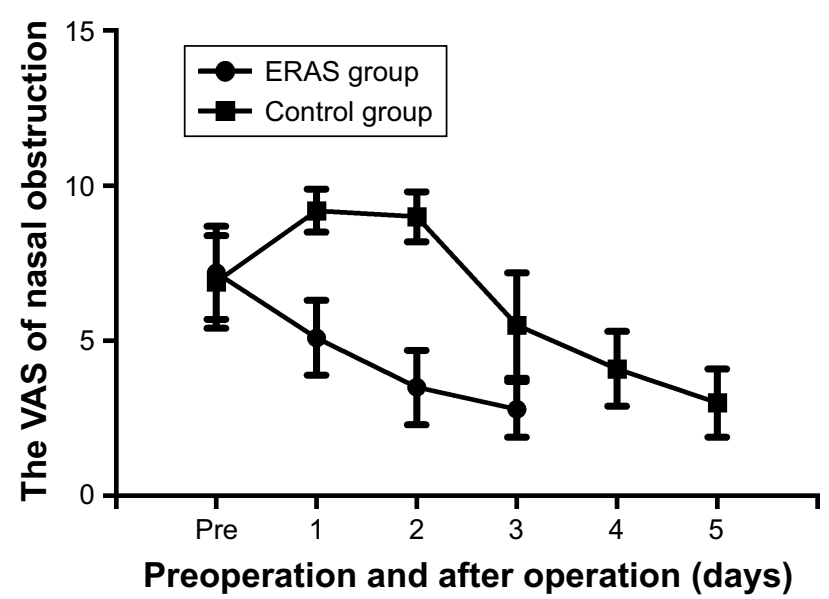

Figure 4 The VAS of nasal obstruction in ERAS group was lower than the control group.

Abbreviations: ERAS, enhanced recovery after surgery; VAS, visual analog symptom score. pain and high cost. ${ }^{14}$ In our study, ERAS was capable of preventing these problems while improving the patient quality of life postsurgery.

In traditional nasal endoscopic septoplasty, nasal cavity filling was always applied postoperatively to prevent postoperative bleeding. However, the application of nasal filling is highly dependent on physician experience to prevent bleeding and hematoma while stabilizing the septum, not to mention that it can be the source of mucosa damage and can increase pain, cost, and complications. ${ }^{15}$ Moreover, traditional surgical procedures caused patients to be more prone to anxiety and poor medical experience before surgery (Figure 3 ). To address this, the systemic therapy of ERAS which includes the use of intraoperative trans-septal suture supplemented with pain control removed the need for nasal filling and avoided the tampering of the common meatus. The most commonly used material in septal suture surgery is the vircryl 4-0 line, which is a nontoxic and nonirritant material that can be absorbed. The intraoperative treatment of patients in the ERAS group utilized trans-septal sutures for nasal septum, which can fix the nasal septum cartilage and repair torn mucosa. Furthermore, the nasal septal suture not only can prevent septal hemorrhage and hematoma but also lessen patient pain and anxiety during nasal packing removal. The choice of the suture was made based on a randomized controlled clinical study by Awan and Iqbal, ${ }^{8}$ which showed that nasal septal suture was better than nasal filling in reducing postoperative pain, but there was no difference in the incidence of postoperative complications. Moreover, Hari et $\mathrm{al}^{9}$ also applied uninterrupted suture technique instead of interrupted suture technique to deal with the septum, which can better protect torn local mucosa and prevent postoperative complications such as bleeding and septal hematoma. Postoperative patients can avoid the direct nasal pain source caused by the filling, and other indirect postoperative discomfort such as dry mouth, inappetence, and difficulty sleeping caused by mouth 


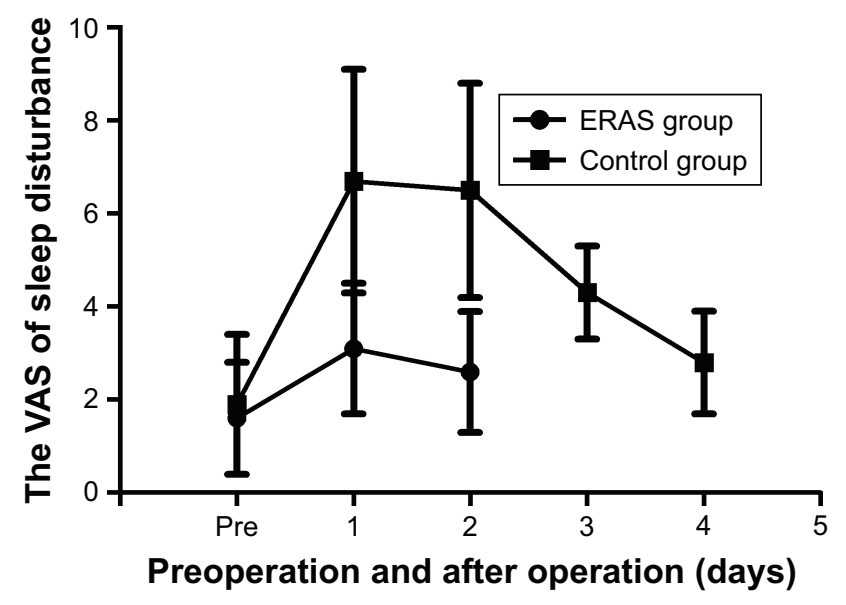

Figure $\mathbf{5}$ The VAS of sleep disturbance in ERAS group was lower than the control group.

Abbreviations: ERAS, enhanced recovery after surgery; VAS, visual analog symptom score.

breathing. Additionally, eliminating the use of nasal filling can also prevent the pain, bleeding, and nasal mucosa damage from its removal 2 days postoperation. The prevention and pain management postoperation through the application of ERAS can thus relieve postoperative patient anxiety and improve overall postoperative experience (Figures 3-6).

With the modernization of medical practice comes the rising cost in medical expenses. As such, our study showed that the application of ERAS can shorten the time of hospitalization and therefore reduce the total cost of hospitalization and medical procedures. The finding on ERAS application is in line with the current health care state where medical resources are barely meeting the rising demands and a new health care plan need to be devised to control medical and medical operations cost while improving patient

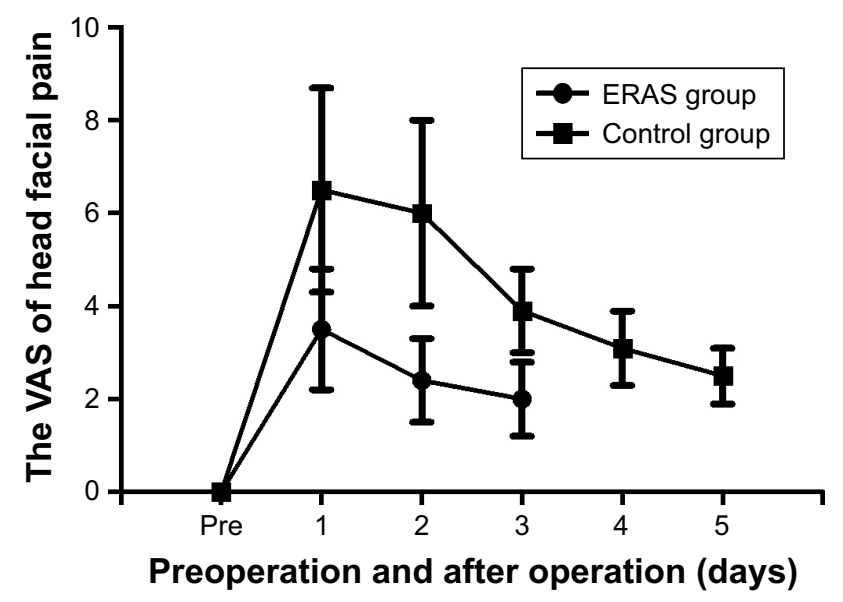

Figure 6 The VAS of head facial pain in ERAS group was lower than the control group.

Abbreviations: ERAS, enhanced recovery after surgery; VAS, visual analog symptom score. experience and their quality of life. Additionally, application of ERAS can improve physician-patient communications to improve compliance and reduce other negative impacts in the treatment processes, which also indirectly increase patient satisfaction as well as confidence in the health care scene. Therefore, the first application of ERAS in the field of otolaryngology yielded a promising outcome so that more large-scale implementation can happen in the future.

\section{Conclusion}

Our study indicated ERAS application can reduce hospital charges and postoperative pain in septoplasty, thereby improving patient quality of life and hospital expenses at the same time.

\section{Acknowledgment}

This study was supported by the Science and Technology Project of Guangdong Province (no 2016A020215030, and 2017A020215180) and the Science and Technology Foundation of Zhuhai (20161027F060002).

\section{Disclosure}

The authors report no conflicts of interest in this work.

\section{References}

1. Most SP, Rudy SF. Septoplasty: Basic and Advanced Techniques. Facial Plast Surg Clin North Am. 2017;25(2):161-169.

2. Wang WW, Dong BC. Comparison on effectiveness of trans-septal suturing versus nasal packing after septoplasty: a systematic review and meta-analysis. Eur Arch Otorhinolaryngol. 2017;274(11):3915-3925.

3. Fearon KC, Ljungqvist O, von Meyenfeldt M, et al. Enhanced recovery after surgery: a consensus review of clinical care for patients undergoing colonic resection. Clin Nutr. 2005;24(3):466-477.

4. Ljungqvist O, Scott M, Fearon KC. Enhanced Recovery After Surgery: A Review. JAMA Surg. 2017;152(3):292-298.

5. Ljungqvist O. ERAS-enhanced recovery after surgery: moving evidence-based perioperative care to practice. JPEN J Parenter Enteral Nutr. 2014;38(5):559-566.

6. Walter CJ, Collin J, Dumville JC, Drew PJ, Monson JR. Enhanced recovery in colorectal resections: a systematic review and meta-analysis. Colorectal Dis. 2009;11(4):344-353.

7. Rasmussen S, Kristensen BB, Foldager S, Myhrmann L, Kehlet H. Accelerated recovery program after hip fracture surgery. Ugeskr Laeger. 2002;165(1):29-33.

8. Awan MS, Iqbal M. Nasal packing after septoplasty: a randomized comparison of packing versus no packing in 88 patients. Ear Nose Throat J. 2008;87(11):624-627.

9. Hari C, Marnane C, Wormald PJ. Quilting sutures for nasal septum. J Laryngol Otol. 2008;122(5):522-523.

10. Kim YH, Kim BJ, Bang KH, Hwang Y, Jang TY. Septoplasty improves life quality related to allergy in patients with septal deviation and allergic rhinitis. Otolaryngol Head Neck Surg. 2011;145(6):910-914.

11. Morgan KA, Lancaster WP, Walters ML, et al. Enhanced recovery after surgery protocols are valuable in Pancreas Surgery Patients. $J$ Am Coll Surg. 2016;222(4):658-664.

12. Fidan T, Fidan V, Ak M, Sütbeyaz Y. Neuropsychiatric symptoms, quality of sleep and quality of life in patients diagnosed with nasal septal deviation. Kulak Burun Bogaz Ihtis Derg. 2011;21(6):312-317. 
13. Mays S. Nasal septal deviation in a mediaeval population. Am J Phys Anthropol. 2012;148(3):319-326.

14. Hong CJ, Monteiro E, Badhiwala J, et al. Open versus endoscopic septoplasty techniques: A systematic review and meta-analysis. Am J Rhinol Allergy. 2016;30(6):436-442.
15. Dadgarnia M, Meybodian M, Karbasi A, et al. Comparing nasal packing with trans-septal suturing following septoplasty: a randomized clinical trial. Eur Arch Otorhinolaryngol. 2017;274(9):3513-3518.

Therapeutics and Clinical Risk Management

\section{Publish your work in this journal}

Therapeutics and Clinical Risk Management is an international, peerreviewed journal of clinical therapeutics and risk management, focusing on concise rapid reporting of clinical studies in all therapeutic areas, outcomes, safety, and programs for the effective, safe, and sustained use of medicines. This journal is indexed on PubMed Central, CAS,
EMBase, Scopus and the Elsevier Bibliographic databases. The manuscript management system is completely online and includes a very quick and fair peer-review system, which is all easy to use. Visit http://www.dovepress.com/testimonials.php to read real quotes from published authors.

Submit your manuscript here: http://www.dovepress.com/therapeutics-and-clinical-risk-management-journal 